\title{
The high-energy Sun - probing the origins of particle acceleration on our nearest star
}

\author{
S. A Matthews ${ }^{1}$ D $\cdot$ H. A. S. Reid ${ }^{1}$ D D. Baker ${ }^{1}$ - D. S. Bloomfield ${ }^{2} \mathbb{D}$.

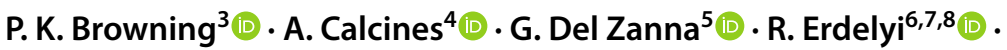 \\ L. Fletcher 9,10 (I) I. G. Hannah ${ }^{9}$ (D) N. Jeffrey ${ }^{2}$ (D) L. Klein ${ }^{11} \cdot$ S. Krucker ${ }^{12}$. \\ E. Kontar ${ }^{9}$ (D) D. M. Long ${ }^{1}$ (D) A. MacKinnon ${ }^{9}$ - G. Mann ${ }^{13}$. \\ M. Mathioudakis ${ }^{14}(\mathbb{D}) \cdot$ R. Milligan ${ }^{14}$ (D) V. M. Nakariakov ${ }^{15}$ (D)

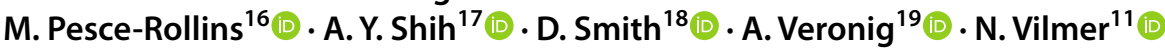

Received: 30 July 2020 / Accepted: 23 September 2021 / Published online: 9 November 2021

(c) The Author(s) 2021

\begin{abstract}
As a frequent and energetic particle accelerator, our Sun provides us with an excellent astrophysical laboratory for understanding the fundamental process of particle acceleration. The exploitation of radiative diagnostics from electrons has shown that acceleration operates on sub-second time scales in a complex magnetic environment, where direct electric fields, wave turbulence, and shock waves all must contribute, although precise details are severely lacking. Ions were assumed to be accelerated in a similar manner to electrons, but $\gamma$-ray imaging confirmed that emission sources are spatially separated from X-ray sources, suggesting distinctly different acceleration mechanisms. Current X-ray and $\gamma$-ray spectroscopy provides only a basic understanding of accelerated particle spectra and the total energy budgets are therefore poorly constrained. Additionally, the recent detection of relativistic ion signatures lasting many hours, without an electron counterpart, is an enigma. We propose a single platform to directly measure the physical conditions present in the energy release sites and the environment in which the particles propagate and deposit their energy. To address this fundamental issue, we set out a suite of dedicated instruments that will probe both electrons and ions simultaneously to observe; high (seconds) temporal resolution photon spectra $(4 \mathrm{keV}-150 \mathrm{MeV})$ with simultaneous imaging (1 keV - $30 \mathrm{MeV})$, polarization measurements (5-1000 keV) and high spatial and temporal resolution imaging spectroscopy in the UV/EUV/SXR (soft X-ray) regimes. These instruments will observe the broad range of radiative signatures produced in the solar atmosphere by accelerated particles.
\end{abstract}

Keywords Particle acceleration $\cdot$ Solar flares $\cdot$ Solar corona $\cdot \gamma$-rays $\cdot$ Hard X-rays

H. A. S. Reid

hamish.reid@ucl.ac.uk

Extended author information available on the last page of the article 


\section{Introduction}

Particle acceleration is a ubiquitous process throughout the Universe, observed in environments as diverse as stellar coronae, active galactic nuclei, the coronae of accretion discs around black holes, the magnetospheres of neutron stars and planetary atmospheres (including our own) interacting with the wind of their star. It operates both in very dynamic and explosive situations and in more steady phenomena where steep gradients, turbulence and instabilities exist. The product of this process, or processes, are energetic particles that fill the Universe, and shape the way in which our own and other Solar Systems work, and what the conditions for the emergence and continuation of life might be.

The Sun is a privileged place to study this fundamental astrophysical problem. It is the only astrophysical setting that allows us to probe particle acceleration by a combination of remote sensing (imaging spectroscopy) and in situ measurements, with time resolution that resolves many of the processes of acceleration and transport. This White Paper introduces a mission concept, SPARK, aimed at making significant advances in understanding particle acceleration and transport.

Energetic particles are seen in nearly every manifestation of magnetic energy conversion, from large flares down to minor explosive events in active regions, and sometimes even the quiescent solar atmosphere. Several decades of hard X-ray (HXR) observations have provided substantial insights into electron acceleration, but the low energy end of the accelerated electron distribution remains poorly constrained, with profound consequences for the flare energy budget. HXR observations have also highlighted how poorly observed the $\gamma$-ray range is, and how little we still know about ion acceleration and its relationship to electron acceleration. FERMI $\gamma$-ray observations hint that proton acceleration in flares and eruptions is very common. RHESSI $\gamma$-ray imaging, possible in only a handful of flares, showed unexpected offsets between the locations of $\gamma$-ray and HXR emission - tantalising evidence that either transport or acceleration for electrons and ions are different. Measurements of ions in space suggest that flare-accelerated ions trapped in the solar atmosphere may have an energy content that is in fact far greater than any other constituent of flare energy. Yet, diagnostics of ions with energies $\leq 1 \mathrm{MeV}$ are few, and those that do exist are rarely sampled by current instrumentation.

The following areas thus represent a continuing significant gap in our understanding of particle acceleration that require new approaches and instrumentation:

1. The Sun as a laboratory for understanding particle acceleration.

2. What is the transition between plasma heating and particle acceleration?

3. What are the processes responsible for ion acceleration, and what is their relationship to electron acceleration processes?

4. How and where are the most energetic particles accelerated on the Sun?

5. What is the role of the magnetic field in determining the onset and evolution of particle acceleration, and what is the role of energy transport effects?

6. $\gamma$-ray emission as a tracer of plasma composition? 
The details of the processes responsible for ion acceleration and their relationship to electron acceleration in particular represent a fundamental gap in our understanding of particle acceleration on the Sun, and there has never been a mission capable of providing the combined spatial, spectral and temporal resolution needed to fill this gap. In the following sections of this White Paper we outline the required measurements to address these areas and two possible mission scenarios that could fill the gaps in our current knowledge.

\section{The Sun as a laboratory for understanding particle acceleration}

At the fundamental level, particle acceleration can occur as a result of particle interactions with DC electric fields, shocks, or plasma turbulence. It is important to acknowledge that while remote sensing observations of the solar atmosphere offer a wealth of diagnostics of the particle acceleration process, they are indirect. Without in-situ measurements of the acceleration region it is difficult to definitively identify the processes at work. However, the range and sophistication of the diagnostics available to us provides the necessary tools to allow us to evaluate different models and rule out those that cannot reproduce the observations.

On the Sun, the commonly held picture is a solar particle accelerator in the corona, where the magnetic pressure is much higher than the gas pressure. Accelerated particles either stream down into the dense atmosphere of the Sun, producing $\mathrm{HXR} / \gamma$-rays, or stream up into the tenuous solar corona and into interplanetary space. However, in confined flares, where particles do not escape to the middle and high corona [1], the acceleration region is likely to be low-lying. Similarly, during solar maximum, the background level of low-energy X-rays significantly increases, likely caused by an increase of low-altitude particle acceleration. Conversely, radio noise storms are signatures of particle acceleration much higher in the corona and can last from hours to days at a time. So, what seems clear is that the Sun accelerates particles in a range of different environments and magnetic topologies. What is much less clear are the properties and location of the acceleration regions and the processes operating within them. In the corona this is in part due to a low density, leading to low intensity EM emission, as well as a lack of diagnostics sampling the high temperatures expected to be present in the region.

The most direct quantitative diagnostics of energetic particles interacting at the Sun come from $\mathrm{HXR} / \gamma$-ray observations. They carry information on electron and ion energy spectra, numbers, energy contents and abundances. While bremsstrahlung X-ray continuum emission provides diagnostic information about energetic electrons, $\gamma$-ray lines from 0.4 to $8 \mathrm{MeV}$ tell us about ions above a few $\mathrm{MeV}$ in energy, and the continuum above $100 \mathrm{MeV}$ yields information about ions $>0.2 \mathrm{GeV} /$ nuc (e.g. [2, 3]. Radio observations also provide diagnostics of energetic electrons. Incoherent gyrosynchrotron emission is due to electrons with energies larger than hundreds of keV, low in the corona, while coherent plasma emission typically provides diagnostics of energetic electrons at a range of altitudes throughout the corona, commonly linked to propagating electrons travelling out from the Sun and through interplanetary space. 

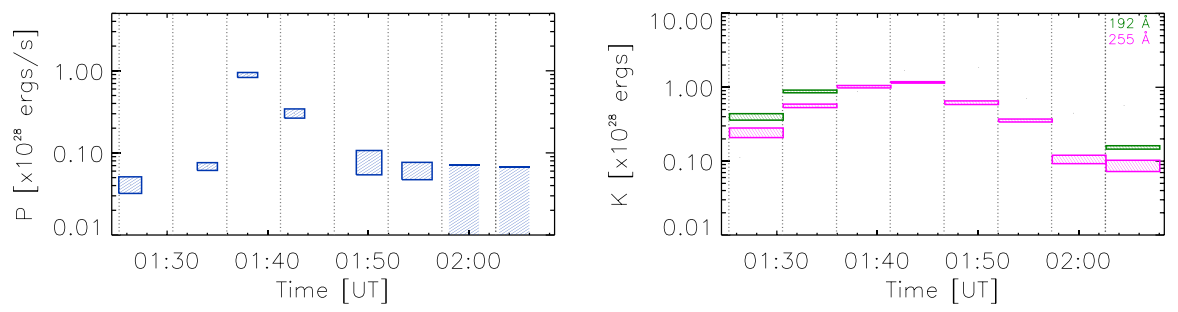

Fig. 1 Left panel: power $\mathrm{P}\left(\mathrm{erg} \mathrm{s}^{-1}\right.$ ) in nonthermal electrons above the low-energy cutoff $\mathrm{E}_{\mathrm{c}}$. Right panel: bulk turbulent kinetic energy K (erg). From Kontar et al. [18]

The X-ray linear polarisation is another key diagnostic of the particle acceleration mechanism, being directly related to the anisotropy of the emitting electron distribution, but the anisotropy is completely unknown in most flare observations. In a simple transport model, often the injected and emitting electrons are assumed to be beamed along the guiding field lines (e.g. [4]). However, if electrons are accelerated by a second-order Fermi process, then their angular distribution might be isotropic (e.g. [5-7]). Ultimately, electron transport through the surrounding solar plasma broadens the electron distribution, increasing the isotropy by collisional or non-collisional scattering (e.g. [8]). Therefore, even if the injected distribution is strongly beamed, the angular distribution of radiating electrons will isotropise on their journey from the corona to the chromosphere. HXR and electron directivity can be studied using statistical flare studies of centre-to-limb variations in flux or spectral index (e.g. $[9,10])$, and simultaneous observations of a single flare with two satellites at different viewing angles. (e.g. [11, 12]), but albedo mirror analysis of strong solar flares [13, 14], and linear X-ray polarization measurements (e.g. [15-17]), are the only methods that can study anisotropy in a single flare using a single instrument.

More indirect diagnostics provide the complementary information that is needed to assess the role of different mechanisms. For example, the profiles of spectral lines formed in the plasma in and around the acceleration region give the possibility to infer the presence of shocks, waves and/or turbulence, which coupled with imaging and spectroscopic measurements of HXR emission can be used to provide evidence for stochastic acceleration. A recent example of this can be seen in Fig. 1 from Kontar at al. [18], which demonstrates the presence of a sufficient reservoir of wave turbulence derived from line broadening measurements (right panel) to account for the non-thermal energy of the accelerated electrons (left panel), lending support to stochastic acceleration models. Other promising techniques include the study of coronal hard X-ray sources. Such events are generally only observed when the luminous $\mathrm{X}$-ray sources from the chromosphere are occulted by the solar disc, which allows the fainter X-ray sources in the corona to be imaged using the indirect methods currently available. There has been much speculation about whether these sources are a direct signature of particle acceleration in the solar corona, but recent observations are hard to explain without invoking particle acceleration. However, the classical model combining particle acceleration in the corona and transport to the HXR and $\gamma$-ray sources in the chromosphere faces a number of unsolved problems [19]. It is 
still not clear whether these sources are the main source of accelerated particles or whether there are additional acceleration sites elsewhere in the atmosphere.

Another promising candidate in this respect is the termination shock (TS) that is predicted to be produced by reconnection outflows colliding with dense plasma in closed post reconnection magnetic loops. Some previous observations of coronal HXR sources have indicated the presence of 'loop-top' sources consistent with the presence of accelerated electrons at or above the top of flare loops, where one would expect the TS to be located. Mann et al. [20] demonstrated that these fast-mode shocks are capable of accelerating electrons to observed levels, and recent work by Chen et al. [21] combined observations of a TS observed in the radio, with imaging in the EUV and X-ray to link the TS to the reconnection outflow and characteristic geometry and dynamics of a cusp-shaped reconnection scenario. Supporting simulations were able to reproduce the observed TS characteristics well, suggesting that, in that case, a termination shock played a significant role in the acceleration of energetic electrons. More recent work by Polito et al. [22] using spectroscopic measurements from the Interface Region Imaging Spectrograph (IRIS, [23]) of the Fe XXI line indicates the presence of red- and blue-shifts of up to $200 \mathrm{~km} / \mathrm{s}$ that are spatially simultaneous with 30-70 keV hard X-ray sources, citing this as potential evidence of acceleration at a termination shock.

All of the above discussion relates to diagnostics of accelerated electrons, but we still know very little about proton and ion acceleration processes, with the majority of our understanding based on spatially unresolved observations from the Solar Maximum Mission (SMM) and the Compton Gamma-ray Observatory (CGRO) which were operational in the 1980 and 1990 s. More recent observations from FERMI and RHESSI strongly indicate differences in the electron and ion acceleration and transport processes that require further investigation. In addition, although we have reasonable diagnostics of $>1 \mathrm{MeV}$ ions, we have few probes of the sub$\mathrm{MeV}$ component. This could imply a very substantial under-estimate of the total energy budget for ions, with implications for viable acceleration processes. New developments in $\gamma$-ray imaging and spectroscopy now offer the opportunity to probe this poorly explored regime of the spectrum and to make major advances. In addition, they open a new field of comparative studies with in situ measurements that commonly address protons and ions.

\section{What is the transition between plasma heating and particle acceleration?}

In principle, the collisional thick target model allows us to infer the properties of the underlying accelerated electron spectrum from the parameterization of the HXR spectrum, and within this framework it is seen to generally be the case that the spectrum is well characterised by a power-law or broken power-law above a low energy cut-off. This low energy cut-off is critical for constraining the total energy contained in the non-thermal electron distribution and yet it, and the transition from thermal to non-thermal regimes, remains poorly constrained (e.g. [24, 25]). 
However, the direct consequence of heating is thermal emission. It is known (cf. [26]) that the thermal emission that most closely represents the shape of the heating function is in the 10-15 MK range, and the best diagnostics of this plasma are either in the SXR or HXR (see [27]). In both cases spatially resolved spectroscopy is unavailable. However, such observations offer great discovery potential.

The best diagnostics to directly measure electron temperatures, study non-equilibrium ionization and non-thermal electron distributions involve satellite lines and are around 1-5 $\AA$, a region studied with Bragg Crystal Spectrometers on board the P78-1, Hinotori, Solar Maximum Mission, Yohkoh and CORONAS-F missions, but without spatial resolution. The most interesting features (e.g. blue-shifts, excess line broadening) were observed during the impulsive phase, when signals were low, so an instrument with a large collecting area is needed. Gabriel and Phillips [28] demonstrated theoretically that a significant presence of electrons in the high-energy tail of the electron energy distribution would result in an enhancement of resonance lines relative to satellite lines in the X-ray spectrum, and work by Seely et al. [29], and more recently by Dzifcáková et al. [30] found evidence that such distributions are present, but using spatially unresolved observations (with the SOLFLEX and RESIK crystal spectrometers). In order to make further progress spatially resolved observations are crucial. A significant improvement could come from the use of grazing incidence focusing optics (as in FOXSI: [31], or MaGIXS: [32]), combined with X-ray microcalorimeters (cf. [33]).

Additionally, the SXR spectral region (90-150 $)$ ) contains the bright 2-2 transitions from several ionization stages of Iron, from Fe XVIII to Fe XXIII. These lines were observed in 1969 by OSO-5 [34] and later by SDO/EVE without spatial resolution, and provide excellent diagnostics of 5-12 MK plasma [35], including electron densities, unavailable at other wavelengths. Indeed, there are no spatially resolved measurements of electron density in $10 \mathrm{MK}$ plasma, despite the fact that such measurements are key if we want to understand the plasma response to heating, as well as probe for the presence of turbulence and plasma compression in the acceleration region.

\section{What are the processes responsible for ion acceleration, and what is their relationship to electron acceleration?}

From the analysis of HXR observations we know that non-thermal electrons carry a significant fraction of the energy released during a flare [36, 37], but we have only a vague idea how this compares with the energy transferred by protons and heavy ions. HXR/ $\gamma$-ray observations obtained with high spectral resolution enable detailed analysis of the bremsstrahlung continuum and the resolution of individual $\gamma$-ray lines. In the HXR domain bremsstrahlung spectra at high resolution can be directly inverted to get the effective mean electron flux spectrum in the source (e.g. [38]). This quantity is the electron spectrum that would be required to observe the photon spectrum in a homogeneous source and is the only quantity that can be derived from the photon spectrum without making any assumption about the transport of electrons between acceleration and emitting sites. The electron flux spectrum is the 
Fig. 2 Overlay of the $50 \%$, $70 \%$, and $90 \%$ contours of $\gamma$-ray images made with RMCs $6+9$ on a TRACE $195 \AA$ image of the October 28 flare. From Hurford et al. [43]

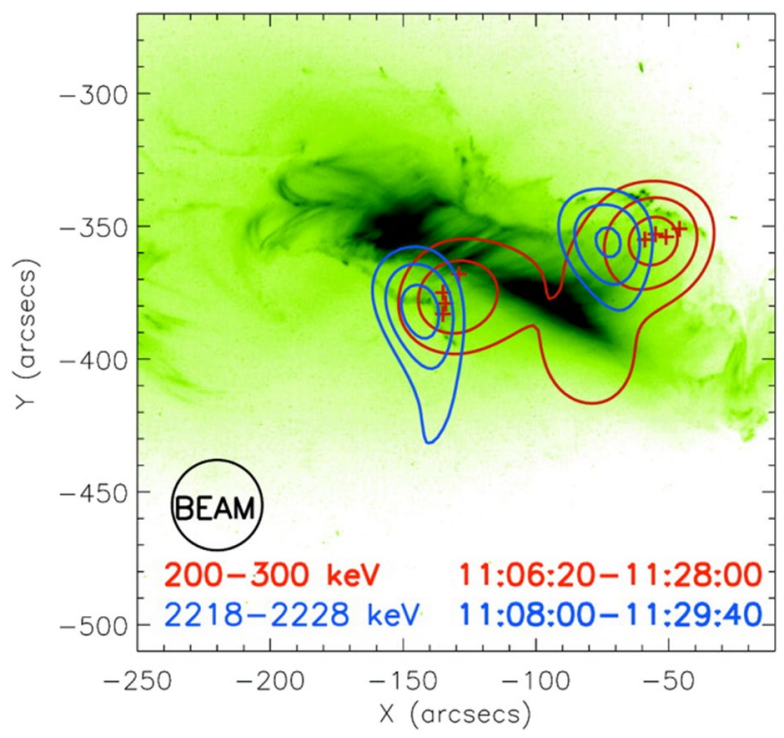

essential quantity to really constrain acceleration models. Such spectra have so far only been obtained for very few $(<10)$ events [39], due to sensitivity constraints.

Past observations show that one cannot assume ions and electrons to be accelerated by the same mechanism, although they show close timing relationships. There are three well-documented types of observations that appear contradictory: observations by SMM and later RHESSI (see [40]) show that the (event-integrated) fluences of electron bremsstrahlung above $300 \mathrm{keV}$ and of the $2.223 \mathrm{MeV}$ line emission, which is indirectly produced by protons above $30 \mathrm{MeV}$, correlate well over the entire observable range, which extends over three orders of magnitude. This shows a close physical relationship between the acceleration processes producing relativistic electrons and ions, indicating that energetic ions could be produced as soon as there is a significant production of energetic electrons above $300 \mathrm{keV}$ [40]. The lack of ion-associated signatures in smaller flares could simply result from a limited sensitivity of previously flown experiments. Individual studies of large events however show differences in the time evolution of electron bremsstrahlung and nuclear line emission [41]. Differences do not only show up in the time evolution: one of the most intriguing results from RHESSI comes from the first/only imaging observations of a $\boldsymbol{\gamma}$-ray line (GRL) event (Fig. 2). The $2.2 \mathrm{MeV}$ neutron capture line location was found to be displaced by $20^{\prime \prime}$ from the centroid of the HXR sources in the 50-100 keV range imaged in the same conditions [42]. The spatial and temporal differences of emissions from electrons and ions are an unanswered challenge to our present understanding of particle acceleration and particle transport in flares.

Imaging in the GRL domain with RHESSI was achieved for only 5 events [3]. Statistically significant displacements between HXR and GRL sources were observed in three of them. In four of the five events where nuclear line emission was imaged by RHESSI, a single unresolved source was observed in the GRL domain. The different electron and ion interaction sites observed in a few events can be interpreted 
as revealing either different electron and ion acceleration sites or showing different transport mechanisms for electrons and ions accelerated in the same site. The explanations for these displacements are, however, still largely unknown. We also so far have only event-integrated images, whereas the full Sun time histories of large flares suggest a strong variation during a given event. The association of images with a higher dynamic range and higher sensitivity in HXR above a few $100 \mathrm{keV}$ and in the GRL domain is critical to reconstruct the path between the regions of acceleration and emission, in order to understand the locations and the conditions for acceleration of electrons and ions in solar flares.

\subsection{What is the total energy content in accelerated ions?}

A rough equipartition in energy between $>20 \mathrm{keV}$ electrons and $>1 \mathrm{MeV}$ ions interacting at the Sun has been found in many events, but flare to flare variations can still be quite large. (see e.g. [3, 37] for a review). These determinations have large uncertainties. In particular, the total ion energy content could be significantly underestimated, since low energy ions (sub MeV) are mostly undetected and the low-energy shape or cut-off of the ion spectrum is completely unknown.

Simnett [44] proposed that protons in fact constitute a far greater fraction of the flare energy budget than electrons, but they have so far eluded detection outside of large $\gamma$-ray events. Orrall and Zirker (1976) described how protons in the 10-1000 keV range could undergo charge exchange with neutral hydrogen atoms in the chromosphere to produce downward streaming non-thermal neutral hydrogen atoms from which Doppler shifted Lyman $\alpha$ emission would be expected in the red wing of the line, with the absence of a corresponding enhancement in the blue wing. Despite the Lyman $\alpha$ line of hydrogen being the brightest emission line in the solar spectrum, high-resolution, spectrally-resolved Lyman $\alpha$ observations during solar flares have not routinely been made since the Skylab era in the $1970 \mathrm{~s}$, and so evidence for low-energy protons remains elusive. Woodgate et al. [45] reported the detection of such an enhancement in the red wing in flaring emission of the star AU Mic, lasting for $3 \mathrm{~s}$ at flare onset that they interpreted as evidence for the existence of a proton beam, while subsequent attempts to detect similar signatures in solar flares, including in He II Ly $\alpha$ line $[46,47]$ have so far not produced a positive result. However, theoretical work by Zhao et al. [48] suggests that the effect may be also be seen in the Ly $\beta$ line, and that it is more pronounced for oblique rather than vertical beams. While low energy proton diagnostics do exist in the $\gamma$-ray regime (e.g. [49]), UV diagnostics offer the additional possibility of spatially resolving the energy deposition sites through imaging spectroscopy. Rastering slit spectroscopy at these wavelengths has been demonstrated with e.g. SUMER and IRIS, and the SPICE spectrometer on Solar Orbiter will observe Ly $\beta$, while spatially unresolved profiles are currently observable with SDO/EVE [50]. However, new developments in integral field spectroscopy (e.g. [51-53]) offer potential for new space instruments capable of simultaneous 2D spectral and spatial observation in the Lyman lines of conjugate flare ribbons and particle deposition sites with high spatial, spectral, and temporal resolution. 


\subsection{What are the spectral characteristics and relative abundances of energetic ions?}

Narrow emission lines produced by accelerated ${ }^{3} \mathrm{He}$ and $\alpha$ particles provide information on the He abundances in the accelerated particles and the ambient solar plasma as well as the ${ }^{3} \mathrm{He}$ abundances [54]. Using SMM/GRS observations, Mandzhavidze et al. [55] found abundance enhancements of the accelerated ${ }^{3} \mathrm{He}$ and $\alpha$ in several $\gamma$-ray flares indicating that particles may be accelerated by the same mechanism (e.g. stochastic acceleration through gyroresonant wave-particle interaction [56]. The implications for accurate determination of ${ }^{3} \mathrm{He}$ abundances goes beyond that of flare physics. ${ }^{3} \mathrm{He}$ is a tracer of nucleosynthesis in the early Universe and estimates of its representative abundance are important constraints for cosmological models. $\gamma$-ray observations of solar flares provide a more direct method of determining ${ }^{3} \mathrm{He}$ in the solar photosphere than from other methods such as solar wind measurements [57].

High spectral resolution in the GRL domain is however essential to constrain the line fluences and to analyse line shapes. Together with detailed calculations of GRL shapes, these observations provide strong constraints on the ratio of accelerated helium with respect to accelerated protons. However, many parameters determine line fluences and line shapes: the angular distribution of interacting ions, spectral index of the energetic ions and $\boldsymbol{\alpha} / \mathrm{p}$ ratio, so that only the combination of line shapes and line fluences can provide strong constraints on those parameters directly linked to particle acceleration and transport models. The number of solar flares for which GRL spectra at high resolution have been obtained is still very small (around five combining RHESSI and INTEGRAL/SPI observations) (see e.g. [41]), and even in these cases the separation of the line emission from the bremsstrahlung and broad line background remains a difficult issue. The relative importance with time of the narrow (produced by the energetic proton and alpha-particle) and broad (produced by the heavier accelerated species) line shapes should also be key evidence for the progress of the energisation process but this sort of study has been achieved for very few events in all existing data [58-60].

\section{How and where are the most energetic particles accelerated at the Sun?}

The most challenging problem in understanding particle acceleration arises at the highest energies. From measurements of escaping particles we know that the Sun is capable of accelerating protons to several $\mathrm{GeV}$ [61], and electrons to nearly $100 \mathrm{MeV}$ [62]. On the Sun, observations of pion-decay radiation from solar flares and direct detections from neutrons provide information on the relativistic ions produced in solar flares (up to several $\mathrm{GeV} / \mathrm{nuc}$ ) and interacting at the Sun. Pion-decay radiation from high-energy protons and neutrons above $100 \mathrm{MeV}$ were first detected aboard SMM/GRS (see reviews by [3, 63]). Before the FERMI launch, all observations of pion decay photons had been related to very large flares (see e.g. [3]).

A major surprise of the Fermi-LAT instrument was the detection of an unexpectedly high number of bursts attributable to pion-decay $\gamma$-rays, i.e. to the interaction 
of ions and protons with energies exceeding $0.2 \mathrm{GeV} / \mathrm{n}$. Even moderate (M-class) flares may show this signature $[64,65]$. Another surprise, although not a discovery $[63,66]$ was the fact that a number of those events had durations of several hours, unlike any other known signature of interacting particles of high energies in the solar atmosphere. It is not clear if these particles are continuously accelerated in the corona [67-69] or whether they are trapped in extended coronal structures [70]. The observation of these phenomena together with sensitive observations at lower energies and with bremsstrahlung from electrons will put these manifestations of the highest energies to which particles are accelerated in solar eruptive events into the context of conventional flare observations.

\subsection{Where are the acceleration regions?}

The few $\gamma$-ray observations of ion interaction sites as shown by images in the 2.2 MeV line suggest that they may be different from those of electrons (Fig. 2). Theoretical models of reconnection-driven acceleration suggest ions and electrons may have the same acceleration regions, but the accelerated particles will be detected in different regions due to being ejected differently from the reconnection site.

However, a standard picture from observations of shock-accelerated particles in interplanetary space is that electrons and protons are generally not accelerated in the same way. A by now popular picture of the interpretation of high-energy longduration $\gamma$-ray events is the acceleration at CME-driven coronal shocks (e.g. [68, 69, $71,72]$ ). The long duration $\gamma$-ray events would then be fundamentally different from more conventional flare-related particle signatures. Localisation of the source region at these high energies with Fermi-LAT is not possible with the precision of imaging observations, however, the localization done for a few events still shows that these high energy emissions are located close to solar active regions (e.g. [64]). Improving the capability to localise these emissions to compare them with flare-related emissions at lower energies would represent major progress. Besides a direct detection similar to Fermi-LAT, which may be technically difficult, an indirect localisation through the identification of correlated signatures at lower photon energies, such as sensitive HXR imaging, could provide completely new insight.

\subsection{Acceleration at large-scale shocks}

Shock waves driven by CMEs are a well-established particle accelerator that is widely invoked to be at the origin of large solar energetic particle (SEP) events in space (e.g., [73]) and of long-lasting $\gamma$-ray emissions in the solar atmosphere (e.g., [69]). While both the shock-accelerated particles and the shock itself can be probed by in situ measurements near $1 \mathrm{AU}$, and will be probed closer to the Sun by Parker Solar Probe and Solar Orbiter, there is no diagnostic so far to do a similar analysis in the corona. This would, however, be crucial to infer commonalities and differences between the processes that lead to energetic particle populations in the corona on the one hand, and SEPs in interplanetary space on the other. New methods of EUV 
analysis, developed using the SWAP instrument onboard Proba-2, which has shown that low coronal features can be observed in the EUV at heights exceeding 1.5 solar radii, albeit following advanced image processing. However, issues remain with directly relating faint features observed on-disc (such as globally propagating shock waves associated with the early evolution of coronal mass ejections) to brighter features observed off-disc (such as the CMEs themselves). The recently proposed COSIE instrument is designed to observe the solar disc and low to middle corona simultaneously using EUV wavelengths (see [74] for the coronal imaging). This enables identification of shock waves propagating in the low corona, and a comparison with energetic particle signatures in HXR and $\gamma$-ray images. This approach will allow substantial progress to be made in understanding the relationship between CMEs, global shock waves and their associated energetic particles.

\section{What is the role of the magnetic field in determining the onset and evolution of particle acceleration and what is the role of energy transport effects?}

Magnetic reconnection - the reorganization of the topology and connectivity of magnetic field lines - is one of the primary means through which magnetic energy can be released, and nonthermal particles can be accelerated (e.g. [75]). In eruptive flares, the close relationship and coupling between the magnetic reconnection in the largescale current sheet formed behind the erupting structure, and the flare energy release and particle acceleration has been quantified in a number of parameters: in terms of a close temporal relation between both processes (e.g., [76, 77]), as well as distinct correlations between the $\mathrm{CME}$ acceleration and various parameters of the accelerated electron spectrum, such as the total number and the spectral hardness [78].

After the initial energy release, the number and species of accelerated particles is determined by the evolution of the field and the local plasma environment. In a strongly magnetised, low-beta plasma a large fraction of the magnetic free energy must be transported away from the immediate reconnection site by kinetic or MHD perturbations. Magnetic field structure and topology play an important role in directing these perturbations and converting their energy to that of accelerated particles by generating parallel and perpendicular shock geometries, providing environments where turbulence can be initiated and trapped [79], trapping energetic particles and generating particular configurations where long-lived acceleration sites can exist and recur. The key link between reconnection and particle acceleration thus involves the coupling between the MHD scale on which the reconnection occurs and kinetic ion and electron dissipation scales. However, this separation represents $10^{5}-10^{6}$ orders of magnitude. The observation of kinetic scales is largely unachievable with current remote sensing techniques, and only starting to become possible with in situ measurements. Consequently, this multi-scale coupling remains poorly understood.

Plasmoid-dominated (or tearing mode) reconnection is one scenario that allows for the natural development of a hierarchy of scales through the fragmentation of the current sheet into magnetic islands (e.g. [80, 81]), self consistently bringing the current sheet to kinetic scales (e.g. [82]). Energetic particle transport theory is the 
favoured approach to solving the large-scale particle acceleration and transport problem in shock acceleration and cosmic-ray transport, and in this scenario the primary acceleration mechanism is due to adiabatic compression [83]. Simulations by $\mathrm{Li}$ et al. [83] of plasmoid reconnection in a low beta plasma found that compression in the reconnection layer can also lead to significant particle acceleration of both electrons and ions, and the formation of power-law spectra. They found that the slope of the spectrum depends on the strength of the guide field, a dependence that has been tested through the observation of the behaviour of HXR and UV emission in the flare ribbons (e.g., [84]). Additionally, the simulations also indicated that the accelerated particles are concentrated in the reconnection outflow and magnetic islands. SXR diagnostics would allow identification of the current sheet and reconnection outflow, together with density variations that may be related to compression in the presence of coalescing islands, while HXR diagnostics would allow identification of the coronal HXRs in relation to these, and HXR and $\gamma$-ray spectroscopy the spectral slope, allowing one to test whether such a scenario is consistent with observations.

An associated question is the nature of quasi-periodic pulsations (QPP) which appear to be a common and possibly even intrinsic feature of both thermal and nonthermal emissions produced by flares (e.g., [85]). Detected periods range from a fraction of a second to several tens of minutes. QPP with properties similar to those observed in the microwave and hard X-ray emission are confidently found even in $\gamma$-rays [86]. The effect of QPP could be linked with magnetohydrodynamic oscillatory processes, or with spontaneous, repetitive reconnection and/or charged particle acceleration, or their combination (e.g., [87]).

Electron transport effects can also be probed through polarisation and anisotropy measurements since they can lead to the measured anisotropy at the hard X-ray (HXR) footpoint differing from the original accelerated distribution. However, the presence of non-collisional transport effects such as turbulent scattering (e.g. [8, $88,89]$ ), acting on shorter timescales than collisional scattering, will isotropise any electron anisotropy to a greater extent and have a greater effect on higher electron energies, compared to just collisional scattering in the corona. Using knowledge of the flaring plasma properties from complementary SXR flux and (E)UV observations, it is possible to determine the presence of turbulent scattering and the average properties of the scattering even without imaging information, by comparing with realistic transport simulations.

\section{7 p-ray emission as a tracer of plasma composition?}

Element abundance signatures have long been used as tracers of physical processes throughout astrophysics and the benchmark reference for all cosmic applications is the solar chemical composition. Studies of plasma composition in solar flares show considerable variability in elemental abundances, apparently depending on the emission lines and instruments used, the atomic data used at the time of the measurements, and temperature effects (e.g., [90-94]). Spatially resolved flare observations from Hinode/EIS show that the first ionization potential (FIP) bias varies from $\sim 3+$ in the post-flare loops to unfractionated plasma in the loop footpoints for an 
X8 flare [95] while enhanced FIP bias is found in the current sheet of the same limb flare [96].

The width of $\gamma$-ray spectral lines produced from nuclear de-excitation also provides information on abundances since the strength of narrow lines depends largely on the abundances of the ambient plasma where the lines are produced i.e. $\gamma$-ray production sites in the chromosphere and/or corona (e.g. [58, 97]). Share and Murphy [98] and Ramaty et al. [54] reported that elemental abundances with similar FIPs deduced from narrow-line spectroscopy correlated well with one another. High-FIP elements did not vary from flare to flare, however low-FIP to high-FIP ratios varied by up to a factor of 4 . For a very limited number of flares, the time evolution of the abundances of low-FIP relative to high-FIP has been examined [2, 99] and it was found that the abundances of low-FIP with respect to high-FIP generally increases with time. The studies on abundances deduced from $\gamma$-ray line spectroscopy is however still rare (around 25 events).

\section{Observable parameters and general measurement strategy}

In order to constrain the mechanisms responsible for particle acceleration in the solar atmosphere it is necessary to directly measure the physical conditions present in (a) the energy release and acceleration sites, and (b) the environment in which the accelerated particles propagate and deposit their energy. A dedicated set of instruments on a single platform can achieve this through the combination of imaging and spectroscopy in wavelengths that sample the vast range of energies over which energy release signatures are observed and rapidly probe the fine spatial scales over which particle acceleration signatures are observed. Such measurements can now, in principle, be accomplished by a single payload of dedicated instruments. This would mark a significant advancement over currently available multi-wavelength observations, which relies on different instruments on different platforms, with different science goals and duty cycles, observing the same localised, transient event at the same instant. Milligan and Ireland [100] highlighted the inherent difficulty in coordinating flare observations using multiple spacecraft by showing that over the course of Solar Cycle 24, only 40 flares out of $\sim 7000$ were simultaneously observed by six or more of the available instruments that do not have $100 \%$ duty cycle.

The properties of the spectra of accelerated particles spectra must be probed through the combination of high resolution HXR and $\gamma$-ray spectra. Through the application of imaging in the UV, SXR, HXR, and $\gamma$-ray range, transport effects can be separated from changes in the initial acceleration process. This combination of measurements would provide an unprecedented capability to observe and constrain the particle acceleration processes operating in the solar atmosphere. Determining the location as well as the time-varying spectrum and directivity of accelerated ions and electrons is another key driver for spatially resolved observations in HXR and $\gamma$-rays. Although there have been good reasons to believe that the acceleration region lies in the tenuous corona, while the strongest signatures of the accelerated 
particles are observed in the lower and denser chromosphere, more recent work is beginning to unsettle this picture (e.g. [19]).

Current indirect HXR imaging instruments have notoriously limited dynamic range which makes it impossible for them to simultaneously image X-rays produced by electrons in or close to the acceleration site at the same time as in the sites where the particles precipitate. Because of this, it is currently almost impossible to deconvolve the properties of the acceleration mechanism from the effects of transport effects, although some preliminary work has been achieved on this topic using the imaging spectroscopy capabilities of RHESSI (e.g. [101, 89]) To break through this barrier, an improvement in sensitivity and dynamic range of order 50-100 times is required. This improvement can now be achieved through the use of direct HXR imaging techniques employing grazing-incidence optics.

Similarly, increased sensitivity in $\gamma$-ray imaging spectroscopy is needed to provide a step change in our ability to image $\gamma$-rays, allowing us to produce a time series of images during $\mathrm{X}$ and high $\mathrm{M}$-class flare events. This will provide vital information about the relationship between ion and electron acceleration mechanisms, and strong constraints for current models.

The observable parameters necessary to achieve these science goals must include:

- High (seconds) temporal resolution photon spectra in the energy range $4 \mathrm{keV}$ - $150 \mathrm{MeV}$ : These measurements are the key to establishing the energy distributions of accelerated electrons and protons. Going beyond $100 \mathrm{MeV}$ will let us identify the spectral signatures of relativistic protons and heavier ions, while extending to low energies allows determination of where the non-thermal electron spectra join the thermal background.

- Simultaneous imaging in the range $1 \mathrm{keV}-30 \mathrm{MeV}$ capable of viewing faint and bright X-ray and $\gamma$-ray sources within the same field of view. These observations provide source localisations for both electrons and ions, and contain at low energies the likely transition between non-thermal and thermal electron populations, as a crucial ingredient to investigating the role of non-thermal particles in the energetics of the solar atmosphere. The imaging of nuclear line emissions, together with the extended spectroscopy ensures the substantial improvement of the radiative diagnostics of protons and ions.

- Measurements of the X-ray polarization in the energy range 5-200 keV and $\gamma$-ray imaging polarimetry between 300 and $1000 \mathrm{keV}$ : This long-neglected diagnostic will provide a unique perspective on the angular distribution of high-energy accelerated electrons.

- High spatial $\left(3^{\prime \prime}\right)$ and temporal (10 s) resolution images of the corona in the EUV range $\left(\mathrm{T}=10^{4}-10^{7} \mathrm{~K}\right.$ with a full-Sun field-of-view $(\mathrm{FoV})$ : As already established with STEREO and SDO these reveal the global nature of flares and CMEs, connections between remote magnetic environments, and the extent of particle acceleration sites. They are essential context information for all other measurements.

- High spatial (0.5 - $\left.1^{\prime \prime}\right)$ and temporal resolution UV/SXR imaging spectroscopy (few seconds) covering temperatures between $10^{4}$ and $10^{7} \mathrm{~K}$ : This will provide essential diagnostic information on temperatures, densities, velocities and abun- 
dances in the energy release and particle acceleration sites, including identifying shocks and turbulence, and links between interacting and escaping particle populations.

\subsection{Mission profile}

The science goals outlined in this white paper require access to a broad range of radiative signatures that are produced in the solar atmosphere by accelerated electrons, protons, and ions. In order to meet the measurement objectives there are a range of mission profile options possible, including both single (M-class) to meet the minimum science goals, and multi-spacecraft (L-class) options that would provide additional science particularly related to particle anisotropy and the link between interacting and escaping particles.

\subsection{Single spacecraft option (M-class)}

A single spacecraft mission would include all of the strawman payload instruments outlined below. To ensure that the required sensitivity is achieved by the HXR and $\gamma$-ray instruments the orbit chosen for the primary spacecraft should minimize exposure to the radiation belts and SEP events. This requires a low altitude, low inclination orbit. There are two LEO options that would fall within the constraints of a Soyuz launch. A Sun-synchronous orbit at $600 \mathrm{~km}$ of $98 \mathrm{~min}$ duration would provide excellent data coverage and a good environment for UV instrumentation. Approximately $20 \%$ of this orbit would be in eclipse. It is the highest acceptable orbit of this kind for the X-ray and $\gamma$-ray instrumentation (without additional shielding), but the background will be much higher than for a comparable altitude equatorial orbit. This orbit would be easily achievable with the Soyuz. A second option would be a circular orbit, also at $600 \mathrm{~km}$ and with 10-15 degrees inclination. This would be highly preferable for the high-energy instruments in terms of background. A much larger fraction of the orbit would be in eclipse in this case. With appropriate shielding it may also be feasible to consider an L1 orbit for continuous coverage, although this would alter the launch requirements.

\subsection{Overview of a strawman payload}

Many decades of observing the Sun have shown that co-ordinating observations of transient events such as flares and CMEs with different observing platforms, while achievable, is notoriously difficult. Even with much improved pointing stability and information for space-based observations, co-alignment of datasets also remains subject to errors. Although of relatively minor importance for large-scale phenomena, this can be a serious impediment when studying small-scale processes. A single platform with coordinated instrumentation is essential for producing the best and most comprehensive datasets for studying the underlying processes of particle acceleration. 
A strawman payload that could achieve the required measurements could include the following:

- Super-FOXSI (Super Focusing Optics X-ray Solar Imager): an instrument that builds on the successful heritage of the FOXSI sounding rocket program [31, 102] and uses grazing incidence focusing optics to image directly in HXR over the energy range 4-60 keV with a spectral resolution of $1 \mathrm{keV}$ and a spatial resolution of $\sim 5$ arcsec. Current HXR imaging measurements are obtained through non-focusing rotation modulation collimator (RMC) imaging techniques, such as those used on RHESSI [103]. However, these, and other types of non-focusing imaging methods, have three main limitations that can be overcome by the use of focusing optics: effective area; large non-solar background, and limited dynamic range. The dynamic range for RMC imaging is limited by emission from the entire field of view. For focusing optics, the dynamic range (and resolution) is set by the shape of the point spread function (PSF), thus the sharper the PSF, the better the dynamic range. Current HXR focusing optics already provide a dynamic range $>50$ (indirect imaging methods such as RHESSI $<30$ ), which with appropriate calibration procedures can be increased to $>100$ for events with good statistics. For well-separated sources, this range can be extended to $>1000$.

Super-FOXSI would use similar modules to FOXSI. The effective area would be increased by a longer focal length, by filling the modules with the largest possible number of shells (almost doubling the effective area at $10 \mathrm{keV}$ and adding response to higher energies), by using multilayer coatings and by increasing the diameter of the instrument. FOXSI's Si strip detectors would be replaced with $60 \mu \mathrm{m}$ CdTe strip detectors. CdTe detectors have already been investigated by ISAS for the HXI system on Astro-H and are being used on Solar Orbiter STIX. A long focal length of the order of $10-15 \mathrm{~m}$ is highly preferable in order to improve the high energy response of the telescope. Expandable booms of such length have been successfully used for the NuSTAR mission (a NASA Small Explorer (SMEX) mission) that was launched in 2012.

- GRIPS ( $\gamma$-ray Imager/Polarimeter for Solar flares): GRIPS utilizes 3D positionsensitive germanium detectors and a multi-pitch rotating modulator to provide high spectral resolution and high angular resolution over the range of hard X-rays of $\sim 40 \mathrm{keV}$ to $\gamma$-rays of $\sim 10 \mathrm{MeV}$ [104]. These technologies were tested on a balloon instrument that flew successfully in January 2016 [105], and a spaceborne version would have a grid-spectrometer separation of $\sim 15-20 \mathrm{~m}$, achieved by a deployable boom.

The germanium detectors have a spectral resolution $<1 \%$ FWHM in the $\gamma$-ray range, which is sufficient to measure the Doppler profiles of all nuclear de-excitation lines to constrain the accelerated-ion angular distribution and the alpha/proton ratio in the accelerated population. This excellent spectral resolution also constrains the atmospheric conditions where the 
position-annihilation line is produced and provides high sensitivity to the neutron-capture line and other intrinsically narrow lines.

The multi-pitch rotating modulator, which is a form of coded aperture, enables imaging with an angular resolution of 5 arcsec FWHM for a gridspectrometer separation of $20 \mathrm{~m}$, which matches the angular resolution of Super-FOXSI. Such an angular resolution is comparable to the mean free path of energetic ions at the Sun, and thus would be the intrinsic size scale of the smallest $\gamma$-ray features, including resolving footpoint sources. The multi-pitch rotating modulator provides quasi-continuous coverage in the spatial-frequency domain, which results in high image quality with a pointresponse function that has suppressed sidelobes.

The spectrometer can reconstruct Compton-scatter tracks to determine the approximate source location on the sky and to measure the source polarization. For its high-resolution imaging, GRIPS does not rely on Compton imaging analysis because the resulting angular resolution is broader than the entire Sun, but such analysis is very effective for rejecting any non-solar background that would reduce $\gamma$-ray sensitivity. For measuring $\gamma$-ray polarization, The spectrometer is intrinsically sensitive to measuring $\gamma$-ray polarization above $\sim 150 \mathrm{keV}$ through the azimuthal asymmetry of Compton scattering. The polarization can be mapped as a function of location in the FoV, but this would require a large number of photons.

The spectroscopic performance of GRIPS will be extended to high energies (up to $150 \mathrm{MeV}$ ) by placing an array of high-resolution scintillators (ideally $\mathrm{CeBr} 3$ ) behind the Ge detector array. This will improve the sensitivity to the lines of $\mathrm{C}$ and $\mathrm{O}$ at 4.4 and $6.1 \mathrm{MeV}$ and measure the pion component around $100 \mathrm{MeV}$. The relative intensities of the $\mathrm{C} / \mathrm{O}$ lines, $2.2 \mathrm{MeV}$ neutron capture line (best measured with the Ge array), and the pion component will constrain the spectrum of accelerated protons in the range of a few $\mathrm{MeV}$ to a few hundred $\mathrm{MeV}$.

- A large-FOV EUV spectral imaging instrument similar to the COSIE concept will allow CMEs to be tracked from initiation out to 3 solar radii with unprecedented cadence (tens of seconds) and resolution (3"). COSIE was proposed to NASA as a mission of opportunity to fly on the International Space Station in 2023. The COSIE design employs a simple mirror of $18 \mathrm{~cm}$ diameter (of $1 \mathrm{~m}$ focal length) with an EUV multilayer (centred at 195 Angstroms), to provide EUV images and about 500 times more signal per pixel than one SDO/ AIA EUV band. It can also introduce a grating before the mirror, producing overlappograms in the spectral lines. As the spectral band contains coronal lines formed around 1-2 MK, but also lines formed at 10-15 MK, it will show the locations of the different structures of CME, and how they evolve. In particular, we expect to see signatures of shocks in the hot emission. We have seen wonderful examples of $10 \mathrm{MK}$ emission associated to flux ropes in the SDO/AIA SXR bands, but never saw their evolution outside the AIA FoV. 
The spectra will provide measurements of densities (cf. [106]), temperatures and Doppler shifts.

- UV/EUV/SXR imaging spectrometer: A large FoV imaging spectrometer covering the range $10^{4}-10^{7} \mathrm{~K}$ with arc second resolution would be required to provide measurements of line profiles, temperatures, densities, velocities and chemical abundances. The Japanese Solar-C mission will be a single instrument platform composed of a high resolution $\left(0.4^{\prime \prime}\right)$ EUV/UV spectrometer (EUVST; [107] covering several bands in the EUV/UV through the combination of substrates on a single mirror. A similar approach could form the basis of such an instrument. An alternative approach would be to employ several multilayers (e.g. four with two mirrors/gratings) covering selected spectral regions in the (E)UV and SXR [35], or to employ a multi-slit approach such as that being developed for the Multi-slit Solar Explorer (MUSE; [108]).

\subsection{Multi-spacecraft options (L-class)}

The science output from the single observing platform described above can be significantly enhanced via light-weight multi-spacecraft observations at $1 \mathrm{AU}$. The low mass of the SUPER-FOXSI instrument would allow it to be placed on spacecraft at the L4 and L5 points to provide stereoscopic imaging spectroscopy of HXRs. Multispacecraft measurements of the same event would provide 3D source diagnostics, helping to constrain the total amount of energy contained in flare accelerated electrons. Projection effects would be significantly reduced and the 3D motion of HXR sources with time would enable better understanding of how the magnetic structures that both accelerate and guide accelerated electrons evolve over the duration of the flare.

The multi-spacecraft observations could be further enhanced by a light-weight in situ solar energetic particle (SEP) package for energetic electron and ion detection. Single vantage point observations cannot provide representative information on the intensity, spectrum, and composition of an SEP event, although this has been tacitly assumed over decades. In-situ SEP measurements will provide context particle beam information that can be combined with particle diagnostics at the Sun from $\mathrm{X}$-ray and $\gamma$-ray detections. Particle transport effects can be accounted for using the enhanced knowledge that will be gained from Parker Solar Probe and Solar Orbiter measurements in the interim period before Voyage 2050. Measuring in situ the energetic electrons and ions at multiple points on the same radial distance from the Sun will provide information about how the particles are scattered throughout the heliosphere and further enhance our space weather predictions.

Multi-spacecraft electric and magnetic field measurement would provide a cheap, light-weight way to gain significant contextual information regarding particle transport out from the Sun. Radio observations below the ionospheric cut-off at $10 \mathrm{MHz}$ are signatures of electron beam propagation and CME shock acceleration throughout the solar wind. Multi-point radio analysis can provide triangulation of radio source locations and size estimates. Electric field measurements provide diagnostics of beam-induced Langmuir waves that drive the radio emission. The magnetic field 
direction heavily influences particle beam and measurements at L5 greatly enhance space weather predictions of the geoeffectiveness of coronal mass ejections.

\subsection{Technology challenges}

A single spacecraft capable of meeting the SPARK science requirement could be based heavily on past heritage and the spacecraft and orbit requirements are not challenging. There have now been two successful flights of the FOXSI rocket, and one successful balloon flight of GRIPs, which would form the basis for the development of the $\gamma$-ray imaging spectrometer. Deployable booms have been used successfully on NuSTAR.

The Soft X-ray Spectrometer on Hitomi included the X-ray Calorimeter Spectrometer (XCS) as a key element. Cooling was achieved in this case using supercooled liquid helium and a series of mechanical and magnetic refrigerators. Further development work on microADRs is a leading area within Europe and the SPARK instrumentation suite would definitely benefit from this. European groups also have significant heritage in building (E)UV rastering spectrographs (e.g. SOHO CDS, Hinode EIS, Solar Orbiter SPICE) for imaging spectroscopy. However, recent developments for ground-based facilities (e.g. GRIS on GREGOR, DKIST DL-NIRSP, EST), and a more comprehensive mission concept for Solar C, are pioneering the use of Integral Field Spectrographs (IFS), composed of two subsystems: a subsystem that reformats a 2-D field of view into one or more slits (IFU) and a second subsystem that produces the spectral decomposition (spectrograph). The use of image slicers for the IFU would provide a compact and robust solution that does not introduce polarization, although fibre-fed spectrographs provide a larger FoV. If image slicers are used then accessing the UV spectral range requires the image slicer to be manufactured in glass since better surface roughness results are obtained when polishing, leading to reduced stray light and better optical performance. Currently $100 \mu \mathrm{m}$ thickness mirrors are the thinnest available, but it is likely that developments over the coming years may allow $50 \mu \mathrm{m}$ to be achieved. Further developments of fibre-fed concepts are also likely, so both options should be considered in the first instance.

A multi-spacecraft scenario adds additional orbital and communications complexity, but does not require formation flying.

\subsection{SPARK science in context}

Coverage of the flare spectrum at (E)UV, SXR, HXR and $\gamma$-ray wavelengths can only be achieved by leaving the Earth's atmosphere. We have been fortunate in recent years that RHESSI has provided substantial insights into solar electron acceleration and given us the first imaging observations at $\gamma$-ray wavelengths, which have provided tantalising new clues about ion acceleration and its relationship to electron acceleration. Observations with non-solar missions such as FERMI and NuStar have provided further key measurements of the lowest and highest energy extremes of 
the solar particle spectrum, and with the launch of Solar Orbiter in 2020, STIX will view the Sun in HXRs from closer than ever before.

The launch of Solar Orbiter in 2020 marked the beginning of an exciting new era for solar and heliospheric physics with its unique capabilities for marrying remote sensing and in situ observations from a vantage point close to the Sun and out of the ecliptic plane. The combination of Parker Solar Probe, Solar Orbiter, and the wealth of ground-based radio observatories, e.g. ALMA, LOFAR, EOVSA, MUSER, SKA, $\mathrm{SRH}$, the coming decade will lead to huge advances in our understanding of particle transport. However, these facilities are not well suited to answering detailed questions about the environment and processes operating at the sites of particle acceleration in the solar atmosphere. Particularly with the loss of RHESSI, and an as yet unclear funding route for the FOXSI concept [102], there will be a significant gap in dedicated solar instrumentation capable of making the spectral imaging measurements across the required energy ranges, and at the necessary resolution (spectral, spatial, temporal) that is needed to answer fundamental questions about solar particle acceleration. Beyond the GRIPS balloon, there are no currently funded/planned $\gamma$-ray missions dedicated to solar observation.

On a similar timescale to Solar Orbiter, Solar-C EUVST [107] will provide new insights into how energy can be transported through the layers of the solar atmosphere from the upper chromosphere to the corona, and its spectral range has been chosen to include lines that will sample the hot plasma generated in small-scale reconnection events such as nanoflares. It will be valuable in providing broad temperature coverage of mass motions in flares, and measurements of changing plasma composition (e.g. [109]), as well as providing the first spatially resolved spectral observations of the Lyman $\alpha$ line. The newly-launched GOES-R series of satellites, that will operate beyond 2035, will also provide disc-integrated Lyman $\alpha$ profiles at a crude $1 \AA$ resolution at 10 s cadence. Similarly, the Advanced Space-based Solar Observatory (ASO-S) will also contain a Lyman $\alpha$ Solar Telescope (LST), with both an on-disc and coronal imager. We anticipate that these will provide some new pieces of the puzzle, but they cannot provide the direct particle acceleration diagnostics that we outline here.

\subsubsection{Relationship to ground-based optical telescopes}

In the Voyage 2050 timeframe, the $4 \mathrm{~m}$ Daniel K. Inouye Solar Telescope (DKIST) located in Hawaii will be operating. We also anticipate the planned $4 \mathrm{~m}$ European Solar Telescope (EST) which would be located on the Canary Islands. These telescopes cover from $350 \mathrm{~nm}$ to the near infrared, with DKIST capable of extending to 10 microns, and emphasis measuring photospheric, chromospheric and coronal magnetic fields with high precision, and on capturing the evolution of the atmospheric plasma at high resolution - sub-second timescales and spatial scales of $30 \mathrm{~km}$ (at $500 \mathrm{~nm}$ ). The ground-based facilities will provide the imaging spectroscopy and spectropolarimetry required to gain physical insight into both the large-scale magnetic environment in which particle acceleration and radiation happens, and the smallest spatial and temporal scales in the lower solar atmosphere where much of that energy is dissipated. 
Particle acceleration comes at the expense of energy stored in the magnetic field, so with field measurements informing magnetic extrapolations over a whole active region we can quantify the magnetic free energy released and converted during a flare, examining how the energy changes in space and time in comparison with non-thermal particle populations. On smaller scales around flare ribbons and footpoints, variations in the local field and current at the chromosphere and photosphere (e.g. $[110,111])$ highlight the parts of the global structure most involved in the flare. These changes could themselves lead to particle acceleration in the lower atmosphere. Both large (active region) and small scales of the magnetic field and its variations are accessible, the former by rastering small fields-of-view over an active region. The optical and infrared radiation also play an important diagnostic role since energy deposited by non-thermal particles is radiated by strong optical/ IR lines and continua, with a very close association between chromospheric HXR sources and optical/IR continuum footpoints (e.g. [112, 113]). Line diagnostics can also be used to determine the properties of the chromospheric plasma heated by nonthermals, such as its temperature, density and velocity evolution (e.g. [114]) and the corresponding radiative energy losses. When coupled with radiative hydrodynamic flare simulations, this provides further particle diagnostic information, since the atmospheric response depends on the beam flux, spectral index, and low energy cutoff, thus linking ground-based and space-based non-thermal diagnostics.

\subsubsection{Relationship to ground-based radio telescopes}

We anticipate that a multitude of radio telescopes will be operating during the Voyage 2050 timeframe, supplying context diagnostics of accelerated particles. Solar dedicated telescopes that provide imaging spectroscopy monitoring of the radio Sun, currently include the Extended Owens Valley Array (EOVSA) based in California (1-18 GHz), the Mingantu Spectral Radioheliograph (MUSER) based in Inner Mongolia (0.4-15 GHz), and the Siberian Radioheliograph (SRH) based in Russia (4-8 $\mathrm{GHz}$ ). Moreover, intermittent high-resolution imaging spectroscopy is observed using astrophysical radio telescopes around the globe including the Low Frequency Array (LOFAR), based in Europe (0.01-0.25 GHz), the Murchison Widefield Array (MWA) based in Western Australia (0.07-0.3 GHz), the Karl G. Jansky Very Large Array (JVLA) based in New Mexico (0.074-50 GHz) and the upcoming Square Kilometre Array (SKA), based in Western Australia and South Africa (0.05-12.5 GHz).

Radio signatures map the evolution of electrons energised during solar flares. Low frequency radio $(<1 \mathrm{GHz})$ is generated through the coherent plasma emission mechanism and provides a signature of near-relativistic electrons moving outwards from the Sun, together with diagnostics of the background coronal plasma. The high frequency radio $(>1 \mathrm{GHz}$ ) is generated by gyrosynchrotron emission and provides diagnostics of trapped, relativistic electrons in coronal loops. Both emission processes are highly correlated with HXRs (e.g. [115-117]) and frequently show a strong temporal correlation, with gyrosynchrotron emission being co-spatial with HXR sources and plasma emission appearing higher up in the corona. To help answer the particle acceleration and transport science questions, the relativistic electron diagnostics from high frequency radio can be combined with the 
near-relativistic HXR diagnostics to understand how and where electrons are accelerated over a large range of energies. As well as providing contextual information, there is a significant chance that SUPER-FOXSI can provide co-spatial imaging spectroscopy with the low-frequency radio which will significantly enhance our knowledge about the transport of escaping electrons.

\section{Summary \& conclusions}

The details of particle acceleration processes in astrophysical phenomena remain a fundamental open question. The Sun and the inner heliosphere provide a unique environment in which to study these processes, both through the application of remote sensing diagnostics in the solar atmosphere and in situ measurements of the solar wind and terrestrial magnetosphere. There is no other astrophysical environment in which it is possible to do this on the relevant physical scales. If we are to understand particle acceleration and transport processes in magnetised plasma environments in the distant Universe then understanding them on the Sun must be our starting point. It is also noteworthy that particle acceleration as the result of magnetic reconnection is likely to cause plasma energy loss or physical damage in tokamaks during start-up, and that the dimensionless parameter regime of, e.g. the Mega Amp Spherical Tokamak, plasma in this stage is similar to that in the flaring corona [118].

While great advances have been made in the last decade or so by RHESSI in the area of solar electron acceleration and transport, our understanding of ion acceleration processes is, at best, sketchy, and yet these particles offer us the best opportunity of linking energy release sites in the solar atmosphere with in situ measurements in the solar wind. At the start of its mission, RHESSI provided us with the first $\gamma$-ray images of a solar flare [42]. These five, event integrated images, remain the only $\gamma$-ray images that we have. They indicated that there are statistically significant spatial offsets between the sites of $\gamma$-ray and HXR emission that we have, as yet, been unable to fully explain. Temporally resolved imaging in several lines, coupled with spectroscopy and high dynamic range SXR and HXR imaging and spectroscopy, is the only way to disentangle acceleration and transport effects and identify the acceleration process. FERMI-LAT observations (e.g. [64]) have shown us that even in M-class flares there are a surprisingly high number of relativistic ions and protons, and confirmed that these events may persist for many hours. Where, and how, these particles are accelerated is currently unknown. Similarly, the distribution of $<1 \mathrm{MeV}$ protons is almost completely unknown.

Finally, we note that whilst the high energy mission concept described here would provide thoroughly new observations that would substantially advance our understanding of particle acceleration, one option that could be considered in the context of Voyage 2050 would be the instigation of an L-class opportunity that combines this with one or more missions from the fields of solar, heliospheric, magnetospheric and ionospheric physics to provide a Grand European Heliospheric Observatory that not only addresses major challenges in the Solar-Terrestrial physics discipline but 
provides rapid scientific advances in a holistic approach to science that underpins our European space weather requirements for decades to come.

Funding Sources of funding acknowledged are: R.E acknowledges the support received from the Science and Technology Facilities Council (STFC) UK (grant number ST/M000826/1 at the University of Sheffield). DML is grateful to the Science Technology and Facilities Council for the award of an Ernest Rutherford Fellowship (ST/R003246/1). ROM would like to thank Science and Technologies Facilities Council (UK) for the award of an Ernest Rutherford Fellowship (ST/N004981/1).

Data availability Not applicable.

Code availability Not applicable.

\section{Declarations}

\section{Conflicts of interest/Competing interests Not applicable}

Open Access This article is licensed under a Creative Commons Attribution 4.0 International License, which permits use, sharing, adaptation, distribution and reproduction in any medium or format, as long as you give appropriate credit to the original author(s) and the source, provide a link to the Creative Commons licence, and indicate if changes were made. The images or other third party material in this article are included in the article's Creative Commons licence, unless indicated otherwise in a credit line to the material. If material is not included in the article's Creative Commons licence and your intended use is not permitted by statutory regulation or exceeds the permitted use, you will need to obtain permission directly from the copyright holder. To view a copy of this licence, visit http://creativecommons.org/licen ses/by/4.0/.

\section{References}

1. Klein, K.-L., Trottet, G., Samwel, S., Malandraki, O.: Solar Phys. 269, 309 (2011)

2. Share, G.H., Murphy, R.J.: Geophysical monograph series. In: Gopalswamy, N., Mewaldt, R., Torsti, J. (eds) vol. 165, p. 177 (2006)

3. Vilmer, N., et al.: Space Sci. Rev. 159, 167 (2011)

4. Brown, J.C.: Sol. Phys. 18, 489 (1971)

5. Melrose, D.B.: ApJS 90, 623 (1994)

6. Miller, J.A., Larosa, T.N., Moore, R.L.: ApJ 461, 445 (1996)

7. Petrosian, V.: Space Sci. Rev. 173, 535 (2012)

8. Kontar, E.P., Bian, N.H., Emslie, A.G., Vilmer, N.: ApJ 780, 176 (2014)

9. Ohki, K.-I.: Sol. Phys. 7, 260 (1969)

10. Kašparová, J., Kontar, E.P., Brown, J.C.: A\&A, 466, 705 (2007)

11. Kane, S.R.: Ap\&SS, 75, 163 (1981)

12. Casadei, D., Jeffrey, N.L.S., Kontar, E.P.: A\&A, 606, A2 (2017)

13. Dickson, E.C.M., Kontar, E.P.: Sol. Phys. 284, 405 (2013)

14. Kontar, E.P., Brown, J.C.: ApJ 653, L149 (2006)

15. McConnell, M.L., Smith, D.M., Emslie, A.G., et al.: Adv. Space Res. 34, 462 (2004)

16. Suarez-Garcia, E., Hajdas, W., Wigger, C., et al.: Sol. Phys. 239, 149 (2006)

17. Tindo, I.P., Ivanov, V.D., Mandel'stam, S.L., Shuryghin, A.I.: Sol. Phys. 14, 204 (1970)

18. Kontar, E.P., Perez, J.E., Harra, L.K., Kuznetsov, A.A., Emslie, A.G., Jeffrey, N.L.S., Bian, N.H., Dennis, B.R.: Phys. Rev. Lett. 118(15), 155101 (2017)

19. Fletcher, L., Hudson, H.S.: ApJ 675, 1645 (2008)

20. Mann, G., Aurass, H., Warmuth, A.: Astron. Astrophys. 494, 669 (2009) 
21. Chen, B., Bastian, T.S., Shen, C., Gary, D.E., Krucker, S., Glesener, L.: Science 350(6265), 1238 (2015)

22. Polito, V., Galan, G., Reeves, K.K., Musset, S.: ApJ 865, 161 (2018)

23. De Pontieu, B., et al.: Sol. Phys. 289, 2733 (2014)

24. Veronig, A.M., Brown, J.C., Dennis, B.R., Schwartz, R.A., Sui, L., Tolbert, A.K.: ApJ 621, 482 (2005)

25. Warmuth, A., Mann, G.: A\&A 588, 116 (2016)

26. Parenti, S., Buchlin, E., Cargill, P.J., et al.: ApJ 651, 1219 (2006)

27. Del Zanna, G., Mason, H.E.: Living reviews. Sol. Phys. (2018). https://doi.org/10.1007/ s41116-018-0015-3

28. Gabriel, A., Phillips, K.J.H.: MNRAS 189, 319 (1979)

29. Seely, J.F., Feldman, U., Doschek, G.A., et al.: ApJ 319, 541 (1987)

30. Dzifcáková, et al.:\&nbsp;A\&A 488, 311 (2008)

31. Krucker, S., Christe, S., Glesener, L., et al.: ApJ 793, L32 (2014)

32. Kobayashi, K., Cirtain, J., Golub, L., et al.: Proc. SPIE 7732, 773233 (2010)

33. Laming, J.M., Adams, J., Alexander, D., et al.: ArXiv 1011.4052L (2010)

34. Kastner, S.O., Neupert, W.M., Swartz, M.: ApJ 191, 261 (1974)

35. Del Zanna, G., Andretta, V., Cargill, P.J., et al.: Front. Astron. Space Sci. 8, 33 (2021)

36. Dennis, B.R., Veronig, A., Schwartz, R.A., Sui, L., Tolbert, K.A., Zarro, D.: RHESSI team. Adv. Space Res. 32, 2459 (2003)

37. Emslie, A.G., Dennis, B.R., Shih, A.Y., Chamberlin, P.C., Mewaldt, R.A., Moore, C.S., Share, G.H., Vourlidas, A., Welsch, B.T.: Ap J. 759, 71 (2012)

38. Piana, M., Massone, A.M., Kontar, E.P., Emslie, A.G., Brown, J.C., Schwartz, R.A.: ApJ 595, L127 (2003)

39. Kontar, E.P., Brown, J.C., Emslie, A.G., et al.: Space Sci. Rev. 159, 301 (2011)

40. Shih, A.Y., Lin, R.P., Smith, D.M.: ApJ 698, L152 (2009)

41. Kiener, J., Gros, M., Tatischeff, V., Weidenspointner, G.: A \& A 445, 725 (2006)

42. Hurford, G.J., Schwartz, R.A., Krucker, S., Lin, R.P., Smith, D.M., Vilmer, N.: ApJ 595, L77 (2003)

43. Hurford, G.J., et al.: ApJL 64, 93 (2006)

44. Simnett, G.M.: Space Sci. Rev. 73, 387 (1995)

45. Woodgate, B.E., Robinson, R.D., Carpenter, K.G., Maran, S.P., Shore, S.N.: ApJ 397, L95 (1992)

46. Brosius, J.W.: ApJ 555, 435 (2001)

47. Hudson, H.S., et al.: ApJ 752, 84 (2012)

48. Zhao, X., Fang, C., Henoux, J.C.: A\&A 330, 351 (1998)

49. MacKinnon, A.L.: A\&A, 226, 284 (1989)

50. Brown, S.A., Fletcher, L., Labrosse, N.: A\&A\&nbsp;596, 51 (2016)

51. Calcines, A., et al.: SPIE 9147, id. 91473I (2014a)

52. Calcines, A., Ichimoto, K.: 2014b, 9143, id. 91434\&nbsp;C

53. Calcines, A., Lopez, R.L., Collados, M.: J. Astron. Instrum. 2(1), 1350009 (2014c)

54. Ramaty, R., Mandzhavidze, N., Kozlosky, B.: AIPC 374, 172 (1996)

55. Mandzhavidze, N., Ramaty, R., Kozlovsky, B.: ApJ, 518, 918 (1999)

56. Ramaty, R., Mandzhavidze, N.: AIPC 522, 401 (2000)

57. Hua, X.M., Lingenfelter, R.E.: ApJ 319, 555 (1987)

58. Murphy, R.J., Ramaty, R., Kozlovsky, B., Reames, D.V.: ApJ 371, 793 (1991)

59. Ramaty, R., Mandzhavidze, N., Barat, C., Trottet, G.: ApJ 479, 458 (1997)

60. Trottet, G., Barat, C., Ramaty, R., Vilmer, N., et al.: AIPC 374, 153 (1996)

61. Ryan, J.M., Lockwood, J.A., Debrunner, H.: SSRv 93, 35 (2000)

62. Moses, D., Droege, W., Meyer, P., Evenson, P.: ApJ 346, 523 (1989)

63. Chupp, E.L., Ryan, J.M.: RAA 9, 11 (2009)

64. Ackermann, M., et al.: ApJ 787, 15 (2014)

65. Ajello, M., et al.: ApJ 789, 20 (2014)

66. Kanbach, G., Bertsch, D.L., Fichtel, C.E.: A\&AS 97, 349 (1993)

67. Omodei, N., et al.: ApJL 865, L7 (2018)

68. Plotnikov, I., Rouillard, A.P., Share, G.H.: A\&A 608, 43 (2017)

69. Share, G.H., Murphy, R.J., White, S.M., et al.: ApJ 869, 182 (2018)

70. Mandzhavidze, N., Ramaty, R.: NuPhS 33, 141 (1993)

71. Gopalswamy, N., Mäkelä, P., Yashiro, S., et al.: ApJ 868, 19 (2018) 
72. Jin, M., Petrosian, V., Liu, W. et al.: ApJ 867, 122 (2018)

73. Reames, D.V. SSRv, 3/4, 41 (1999)

74. Del Zanna, G., Raymond, J., Andretta, V., et al.: ApJ 865, 132 (2018)

75. Fu, H.S., Vaivads, A., Khotyaintsev, Y.V., et al.: GeoRL 44, 37 (2017)

76. Temmer, M., Veronig, A., Vršnak, B., et al.: ApJ 673, L95 (2008)

77. Veronig, A.M., Podladchikova, T., Dissauer, K., et al.: ApJ 868, 107 (2018)

78. Berkebile-Stoiser, S., Veronig, A.M., Bein, B.M., et al.: ApK 753, 88 (2012)

79. Larosa, T.N., Moore, R.L., Shore, S.N.: ApJ 425, 856 (1994)

80. Comisso, L., et al.: PhP1 23, 100702 (2016)

81. Shibata, K., Tanuma, S.: EP\&S 53, 473 (2001)

82. Ji, H., Daughton, W.: PhPl 18k1207 J (2011)

83. Li, X., Guo, F., Li, H., Li, S.: ApJ 866, 4L (2018)

84. Qiu, J., Liu, W.J., Hill, N., Kazachenko, M.: ApJ 725, 319 (2010)

85. Nakariakov, V.M., et al.: PPCF 61, 014024 (2019)

86. Nakariakov, V.M., et al.: ApJL 708, L47 (2010)

87. McLaughlin, J.A., et al.: Space Sci. Rev. 214, 45 (2018)

88. Bespalov, P.A., Zaitsev, V.V., Stepanov, A.V.: ApJ 374, 369 (1991)

89. Musset, S., Kontar, E.P., Vilmer, N. A\&A, 610, A6 (2018)

90. Bentley, R.D.: LNP 483, 3B (1997)

91. Dennis, B.R., Phillips, K.J.H., Schwartz, R.A.: ApJ 803, 67 (2015)

92. Fludra, A., Schmelz, J.T.: A\&A 348, 286 (1999)

93. Phillips, K.J.H., Dennis, B.R.: ApJ 748, 52 (2012)

94. Sylwester, J., Kordylewski, Z., Plocieniak, S.: SoPh 290, 3683S (2015)

95. Doschek, G.A., Warren, H.P., Harra, L.K., et al.: ApJ 853, 178 (2018)

96. Warren, H.P., Brooks, D.H., Ugarte-Urra, I., et al.: ApJ 854 (2018)

97. Ramaty, R., Mandzhavidze, Kozlovsky, B., Murphy, R.J.: ApJ 455, L193 (1995)

98. Share, G.H., Murphy, R.J.: ApJ 452, 933 (1995)

99. Murphy, R.J., Share, G.H., Grove, J.E., et al.: ApJ 490, 883 (1997)

100. Milligan, R.O., Ireland, J.: SoPh 293, 18 (2018)

101. Simões, P.J.A., Kontar, E.P.: A\&A 551, 135 (2013)

102. Krucker, S., et al.: ApJ 739, 96 (2011)

103. Lin, R.P., Dennis, B.R., Hurford, G.J., et al.: Sol. Phys. 210, 3 (2002)

104. Shih, A.Y., et al.:\&nbsp;Proc SPIE 8443, 84434H (2012)

105. Duncan, N., et al.:\&nbsp;Proc. SPIE 9905, 99052Q (2016)

106. Winebarger, A., Weber, M., Bethge, C.: ApJ arXiv:1811.08329 (2019)

107. Shimizu, T., et al., 2019, Proceedings of the SPIE, in press

108. De Pontieu, B., Martinez-Sykora, J., Testa, P., et al.: ApJ 888, 3 (2020)

109. Baker, D., et al.: ApJ 875, 35 (2019)

110. Kleint, L.: ApJ 834, 26 (2017)

111. Sun, X., Hoeksama, J.T., Liu, Y., et al.: ApJ 839, 67 (2017)

112. Fletcher, L., Hannah, I.G., Hudson, H.S., Metcalf, T.R.: ApJ 656, 1187 (2007)

113. Penn, M., Krucker, S., Hudson, H.S.: ApJ 819, L30 (2016)

114. Kuridze, D., Mathioudakis, M., Simões, P.J.A., et al.: ApJ 813, 125 (2015)

115. Pick, M., Vilmer, N.: A\&ARv 16, 1 (2008)

116. Reid, H.A.S., Ratcliffe, H.: RAA 14, 773 (2014)

117. White, S.M., Benz, A.O., Christe, S., et al.: SSRv 159, 225 (2011)

118. McClements, K.G., et al.: Plasma Phys. Control Fusion 60, 025013 (2018)

Publisher's Note Springer Nature remains neutral with regard to jurisdictional claims in published maps and institutional affiliations. 


\section{Authors and Affiliations}

S. A Matthews ${ }^{1}$ D H. A. S. Reid ${ }^{1}$ D D. Daker ${ }^{1}$ D D. S. Bloomfield ${ }^{2}$ D . P. K. Browning ${ }^{3}$ (D) A. Calcines ${ }^{4}$ - G. Del Zanna ${ }^{5}$. R. Erdelyi ${ }^{6,7,8}{ }^{(D)}$.

L. Fletcher ${ }^{9,10}$ (D) I. G. Hannah ${ }^{9}$ (D) N. Jeffrey ${ }^{2}$ (D) L. Klein ${ }^{11}$ - S. Krucker ${ }^{12}$.

E. Kontar ${ }^{9}$ (D) D. M. Long ${ }^{1}$ (D) A. MacKinnon ${ }^{9}$ (D) G. Mann ${ }^{13}$ (D)

M. Mathioudakis ${ }^{14}$ D $\cdot$ R. Milligan ${ }^{14}$ D V. M. Nakariakov ${ }^{15}$ (D)

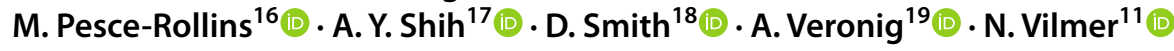

1 UCL, Mullard Space Science Laboratory, Holmbury St Mary, Dorking RH5 6NT, UK

2 Department of Mathematics, Physics and Electrical Engineering, Northumbria University, Newcastle upon Tyne, UK

3 School of Physics \& Astronomy, The University of Manchester, Oxford Road, M13 9PL Manchester, UK

4 Centre for Advanced Instrumentation, Durham University, South Road, DH1 3LE Durham, UK

5 DAMTP, Centre for Mathematical Sciences, University of Cambridge, Wilberforce Road, CB3 0WA Cambridge, UK

6 School of Mathematics \& Statistics, University of Sheffield, Hicks Building, Hounsfield Road, S3 7RH Sheffield, UK

7 Department of Astronomy, Eötvös Loránd University, 1/A Pázmány Péter sétány, H-1117 Budapest, Hungary

8 Gyula Bay Zoltán Solar Observatory (GSO), Hungarian Solar Physics Foundation (HSPF), Petôfi tér 3, Gyula H-5700, Hungary

9 SUPA School of Physics and Astronomy, University of Glasgow, G12 8QQ Glasgow, UK

10 Rosseland Centre for Solar Physics, University of Oslo, P.O.Box 1029, Blindern, Oslo NO-0315, Norway

11 LESIA, Observatoire Paris, 5 Place Jules Janssen, 92190 Meudon, France

12 School of Engineering, FHNW University of Applied Sciences and Arts Northwestern Switzerland, Bahnhofstrasse 6, CH-5210 Windisch, Switzerland

13 Solar Physics Section, Leibniz-Institut für Astrophysik Potsdam, An der Sternwarte 16, D-14482 Potsdam, Germany

14 Astrophysics Research Centre, School of Mathematics and Physics, Queen's University, Belfast BT7 1NN, UK

15 Department of Physics, University of Warwick, Gibbet Hill Road, Coventry CV4 7AL, UK

16 Istituto Nazionale di Fisica Nucleare, Pisa, Italy

17 NASA Goddard Space Flight Centre, Greenbelt, MD 20771, USA

18 Physics Department, UC Santa Cruz, 1156 High Street, 95064 Santa Cruz, CA, USA

19 University of Graz, Universitätsplatz, $58010 \mathrm{Graz}$, Austria 